\title{
Multi-Focus and Multi-Level Techniques for Visualization and Analysis of Networks with Thematic Data
}

\author{
Michele Cossalter, Ole J. Mengshoel and Ted Selker \\ Carnegie Mellon University, Silicon Valley Campus, Moffett Field, CA 94035, U.S.A.
}

\begin{abstract}
Information-rich data sets bring several challenges in the areas of visualization and analysis, even when associated with node-link network visualizations. This paper presents an integration of multi-focus and multi-level techniques that enable interactive, multi-step comparisons in node-link networks. We describe NetEx, a visualization tool that enables users to simultaneously explore different parts of a network and its thematic data, such as time series or conditional probability tables. NetEx, implemented as a Cytoscape plug-in, has been applied to the analysis of electrical power networks, Bayesian networks, and the Enron e-mail repository. In this paper we briefly discuss visualization and analysis of the Enron social network, but focus on data from an electrical power network. Specifically, we demonstrate how NetEx supports the analytical task of electrical power system fault diagnosis. Results from a user study with 25 subjects suggest that NetEx enables more accurate isolation of complex faults compared to an especially designed software tool.
\end{abstract}

Keywords: Network visualization, interactive fault diagnosis, electrical power network, user study, NetEx, Cytoscape

\section{INTRODUCTION}

Networks and graphs are used to model complex systems ranging from engineering artifacts to social relationships. To model and analyze such networks mathematically and computationally, formalisms including graphs (both directed and undirected) and probabilistic graphical models (including Bayesian networks ${ }^{1}$ ) have been introduced and studied extensively in mathematics, statistics, computer science and artificial intelligence.

Electrical power networks play an essential and increasing role in vehicles, including cars, aircraft, and spacecraft. In aerospace, for example, electrical power loads include avionics, propulsion, life support, and thermal management. Unfortunately, electrical power is not without problems. On September 2, 1998, Swissair 111 crashed into the Atlantic Ocean, killing all 229 people on board. It was determined that wires short-circuited and led to an electrical fire. A battery failure occurred on the Mars Global Surveyor on November 2, 2006, ultimately leading to a premature end to its mission. Most likely, a software error caused the battery to overheat due to over-exposure to sunlight. These and similar incidents and accidents emphasize the importance of detecting, isolating, and mitigating faults in electrical power networks. The difficulty of these problem solving tasks increases with the size and complexity of the network, the number and complexity of sensors, the sampling rate of sensors, and the number of and degree of interaction and ambiguity between faults.

The nature of these networks and graphical structures, which are often larger or more complex than analysts can easily keep in their minds, makes interactive visualization software a fundamental tool supporting learning and analysis. Electrical networks, the focus of this paper's user study, have previously been visualized in interesting ways. ${ }^{2,3}$ More generally, there is a rich literature discussing interactive software tools for network visualization. ${ }^{3-8}$ Several techniques and different representations have been developed to improve understandability, ${ }^{2,9-13}$ and enabled an analyst to explore the graph and access low level information. ${ }^{14-21}$ Many researchers have investigated approaches for efficient representation of multi-dimensional data. ${ }^{22-27}$ Multi-focus interaction ${ }^{28-30}$ and the use of multiple coordinated views ${ }^{31-35}$ to facilitate comparison have been studied as well.

To show a graph structure on a computer display, a spatial layout of the node-link diagram must be selected. Depending on several graph characteristics (e.g. size, connectivity) and layout criteria, various algorithms can be applied to accomplish

Further author information: (Please send correspondence to O.J.M.)

M.C.: E-mail: michele.cossalter@gmail.com

O.J.M: E-mail: ole.mengshoel@sv.cmu.edu

T.S.: E-mail: ted.selker@sv.cmu.edu 
this task automatically. ${ }^{36}$ Alternatively, the layout can be created manually. For analysis or debugging, analysts often need to compare and contrast the details (such as time-series) of several nodes that may be rendered close or far away in the network layout. In general, locating the right subset of nodes in the network to closely analyze and compare might be nontrivial. We denote this the multi-focus challenge of visualization. One aspect of the challenge is that for even moderately sized graphs (say, with a 100 nodes or more), the complete graph and all its node labels may not be simultaneously visible on the computer screen. If node labels (or similar annotations on nodes) are not visible, an analyst needs to pan and zoom in to understand what a particular node or set of nodes represent. After panning and zooming in, on the other hand, the overall context and other important nodes located far away in the graph layout may not be visible and could be difficult to remember. Another aspect of this challenge is that real-world graph data, may result in spatial layouts that are quite dense and cluttered. This is the case for the Enron e-mail data set, ${ }^{37}$ for example.

Another research problem, which we denote the multi-level challenge, is the problem of effective and simultaneous visualization of thematic data, such as node attributes, time series, and conditional probability tables, along with the graph structure. Visualizing a large amount of thematic data embedded within the node-link diagram is generally not feasible. Locating the right portion of the data to analyze in depth in order to solve a problem might be like like finding a needle in a haystack. For example, diagnosis of an electrical power network requires checking and comparing time series data from a large number of sensors to look for abnormal behaviors that could flag possible faults. Moreover, only focusing on local information, such as thematic data related to individual nodes, is typically not enough in that an analyst looses context. Global information, i.e. the structure of the network, plays an important role as well in many problem solving tasks. Considering once again the electrical network example, it is essential to understand how power flows from batteries to loads through a distribution network. A key requirement for a network visualization tool is that it should be capable of showing clear connections and relationships between pieces of thematic data and the corresponding graph nodes.

We are interested in interactive visualization and analysis of complex networks where both node-link structure and thematic data are important. The goal of this research is to develop improved visual analytics techniques that meet the multi-focus and multi-level challenges discussed above. Based on the well-understood concept of overview+detail, ${ }^{38}$ our NetEx tool enriches the node-link representation of networks. We leverage existing techniques such as visual style, zooming, and details-on-demand with click-and-drag features. ${ }^{38-40}$ By building on previous work on multi-focus analysis using probes, ${ }^{28,41,42}$ we develop a visualization tool that enriches node-edge representation of networks by providing easy access to thematic data which can not easily be embedded directly in the graph structure. Combining multiple techniques might help orient a deeper analysis of a network. These techniques allow us to simultaneously analyze different aspects and parts of a data set while preserving the layout of the network.

Starting from a network overview, NetEx has been successfully used to fluidly support exploration and comparison of multiple levels of network data. We hypothesize that the techniques integrated in NetEx, while individually relatively simple, are versatile and can be applied to networks of various sizes and types. We have applied NetEx to Bayesian networks with up to 1,000 nodes $^{43,44}$ in addition to the electrical power network ${ }^{45}$ (with around 170 nodes) and social network (with around 150 nodes) discussed in this paper. In this paper we apply NetEx to the diagnosis of faults in a real-world electrical power network, ADAPT. ${ }^{45}$ Results from a user study with 25 subjects suggest that NetEx enables more accurate isolation of complex faults compared to an existing software tool. The generality of this simple approach makes it useful in problem solving situations of "needle(s) in a network," for example when an analyst is looking for one or a few "bugs," something that is an anomaly or not intended. In a biological network, an analyst might be hunting for genes that cause disease or extraordinary behavior. In a social network, like the one we created from the Enron emails, one might be trying to identify incriminating evidence of misconduct.

We first present background and related work in Section 2. Section 3 presents the capabilities of the NetEx software. Starting from a network overview, NetEx analysts can successfully solve complex problems, supported by capabilities for exploration of the network and related thematic data, enabling analysts to compare thematic data for multiple nodes. In Section 3 we discuss NetEx in the context of visualization for example networks, with emphasis on a vehicular electrical power network. ${ }^{45}$ Here, the analytical task, which can be be considered a knowledge crystallization task, ${ }^{46}$ involves examining and comparing time series for a set of sensors (voltage, current, temperature, etc.) in order to diagnose which components of the network broke and at what time it happened. Section 5 reports on a user study with 25 subjects in which two tools, NetEx and Intelliviz, were compared. Our results suggest that NetEx improves the accuracy of complex problem solving task compared to Intelliviz, ${ }^{*}$ a custom software tool that has been productively used to visualize several NASA

\footnotetext{
*http://www.stottlerhenke.com/projects/intelliviz/
} 
data sets, including data from the power network we study here. In Section 6 we conclude and outline future work.

\section{BACKGROUND}

Appropriate visualization techniques might speed and simplify the analysis and exploration of complex datasets, such as electrical power networks. Some dimensions of heterogeneous data may be naturally represented in the form of a (weighted) graph and visualized as node-link diagrams. A graph $G=(\mathbf{V}, \mathbf{E})$ consists of a set of nodes $\mathbf{V}$ and a collection of pairs of nodes called edges $\mathbf{E}=\{(X, Y) \mid X, Y \in \mathbf{V}\}$. A weighted graph $(G, W)$ is obtained by adding a weighting function $W: \mathbf{E} \rightarrow \mathbb{R}^{+}$. Heterogeneous datasets also include other dimensions of data, which in this paper will be referred to as thematic data $D$, that are not directly exposed in the graph structure. The nature of thematic data $D$ varies; examples include time series of physical measurements, as it is for ADAPT, or the textual contents of e-mails or tweets in social networks. The entire network dataset can be described as a tuple $N=(G, W, D)$.

\subsection{Graph Navigation and Exploration}

Several approaches have been developed to visually explore large graphs. Zooming and panning of the graph representation allow users to switch between overview and detailed representations. ${ }^{38}$ More sophisticated interactive graph visualizations provide an integrated representation of details and overview. Research in this area includes the work on subgraph discovery

by Faloutsos et al., ${ }^{14}$ the interaction techniques for subgraph selection and manipulation by McGuffin and Jurisica, ${ }^{16}$ the work on sigma lenses by Pietriga et al., ${ }^{17}$ and research on fisheye view by Schaffer et al. ${ }^{18}$ and Gansner et al. ${ }^{15}$ Relevant work on visual exploration of large graphs also includes the combination of semantic and geometric distortions in the visualization system by van Ham and van Wijk, ${ }^{19}$ the cross-zooming approach by Wong et al., ${ }^{21}$ and the "search, show context, expand on demand" model by van Ham and Perer. ${ }^{20}$ Multiple coordinated views, supporting investigation with distinct views showing different aspects of the same conceptual entity, have been used as well. ${ }^{31,32,35,47}$

NetEx integrates some of the above techniques—especially zooming, panning, and multiple coordinated views—with interactive exploration across multiple node foci.

\subsection{Multi-level and Multi-dimensional Data}

While much information can be encoded in a node-link structure, visualizations have difficulty simultaneously showing underlying thematic data related to nodes. Significant thematic data, such as time series, categorization of messages or the main topics discussed in e-mails, is very hard to visualize as part of a node-link representation, especially when the network is densely connected. Techniques for multidimensional data analysis and comparison have been investigated by Lee et al., ${ }^{23}$ Weaver, ${ }^{27}$ and Lex et al. ${ }^{24}$ Also, several multivariate network visualization systems have been developed, such as PivotGraph ${ }^{26}$ GraphDice, ${ }^{22}$ and FlowVizMenu. ${ }^{25}$ The Action Science Explorer ${ }^{48,49}$ integrates visualization, statistics, and text analytics in a powerful node-arc visualization of scientific citation networks.

Building on work that displays thematic data such as texts, statistics, or time-series, NetEx enables simultaneous display of thematic data for interesting nodes in various parts of a network.

\subsection{Multi-focus and Multi-point Interaction}

Selection of multiple nodes, separated by many links, for detailed comparison might help analysis of graph structures related to large and complex datasets. Ware and Lewis ${ }^{41}$ were concerned with simultaneously zooming in on different parts of an image using several zoom windows. Each window is connected to a zoomed-in patch in a base image by means of probes in the form of straight, solid lines. Shoemaker and Gutwin ${ }^{30}$ further investigated techniques to support multipoint interaction. Also, multi-focus analysis has been studied by Butkiewicz et al., ${ }^{28}$ who describe probe interfaces for geospatial visualization, and Elmqvist et al., ${ }^{29}$ who exploit space folding to guarantee visibility of multiple focus regions. Probes have also been used to connect video playback windows with an overview of a video collection. ${ }^{42}$ Research has also shown the benefit of linking, as opposed to highlighting, in visual data analysis tasks. ${ }^{50}$

It is the explicit visual linking of such thematic data across different parts of a network that we think becomes especially interesting for interactive network exploration as provided by NetEx. 


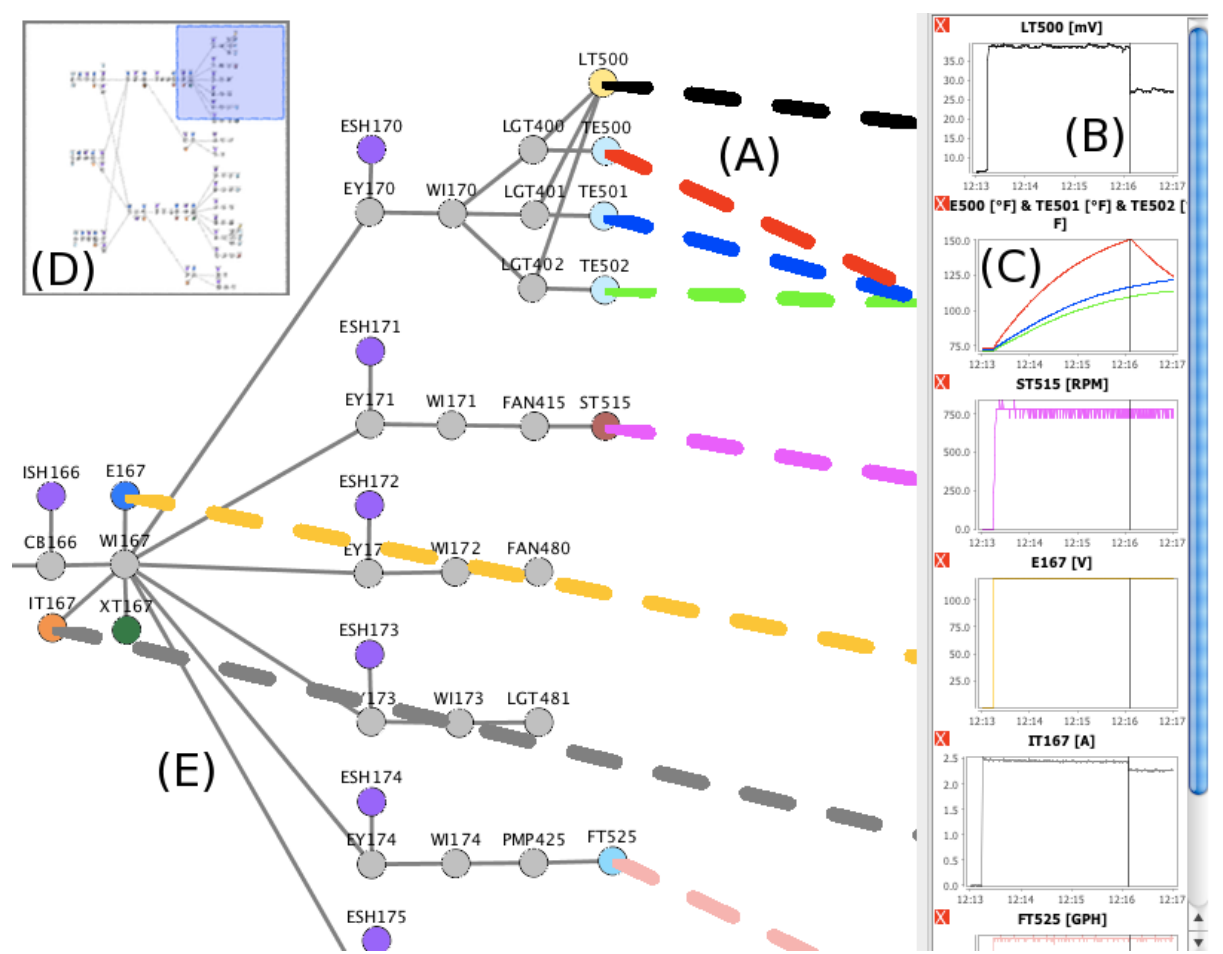

Figure 1. Network visualization with NetEx. Anchoring the thematic data to the network view with dashed bubbles (A) allows low-level focused analysis and comparison. Thematic data boxes (B) can be stacked in a panel alongside of the detailed view (E). A scrollbar allows the panel to host many boxes containing time-series plots. A trace-line is displayed across all data box plots to facilitate comparisons. Data from different nodes (in this case TE500, TE501, and TE502) can also be merged into a single box just by dragging a box and dropping it over another one (C). The overview window (D) prevents the analyst from losing the complete picture of the network.

\subsection{Graph Readability and Filtering}

Several techniques have been investigated to address the problems of density and clutter sometimes associated with nodelink visualizations, e.g. the hyperbolic browser by Lamping et al., ${ }^{11}$ the flow map layout by Phan et al., ${ }^{12}$ EdgeLens by Wong et al., ${ }^{13}$ and Holten's hierarchical edge bundling. ${ }^{10}$ Dynamic filtering to hide the least interesting edges, as well as using opacity to emphasize important edges while keeping all edges visible are other typical solutions to clutter. ${ }^{36}$ Overbye and $W_{e b e r}{ }^{2}$ have investigated different methods for visualization of electric power systems, including animation, dynamic device sizing, contouring, data aggregation and 3D views. Also, a very compact representation of graphs can be obtained by replacing traditional node-link diagrams with adjacency matrices or hybrid representations, e.g. NodeTrix by Henry et al. ${ }^{9}$ However, in an adjacency matrix one may loose some structure that can be easily seen in node-link diagrams.

NetEx could be extended with the above readability, filtering, and clutter-reduction techniques; adding to them the idea of interactively evaluating thematic data for interesting nodes in various parts of a network.

\section{NETWORK VISUALIZATION AND ANALYSIS WITH NETEX}

We describe a multi-focus multi-level visualization approach, implemented in a software tool NetEx that supports integrated network analysis and interactive visualization. NetEx gives the analyst a familiar graph view with modified layout to focus on multiple parts simultaneously using a distinct bubble attachment to other visually associated views. We integrate existing techniques ${ }^{28,31,38}$ to improve multi-concept combination comparisons. The visualization technique considers overview+detail as well as focus+context, in a seamless fashion. The visual representations can be zoomed and panned at all times for overview+detail and provide enhanced interactive focus+context techniques on demand.

$\mathrm{NetEx}^{43,44}$ is an interactive visualization tool for integrated network analysis, implemented as a Cytoscape ${ }^{51}$ plug-in. It gives the analyst a familiar graph view while enabling focus on multiple parts of a network simultaneously, distinguishing 
data at different representation levels with stylized "thinking bubble" links, taken from the thought bubbles found in cartoon strips. These bubble links support both multi-level and multi-focus analysis, which we now discuss.

\subsection{Multi-level Network Analysis: Overview, Detailed View, and Thematic Data}

Figure 1 shows a screen-shot of NetEx. The network overview is always visible (Figure 1(D)). The detail view allows comparison of semantics not brought out in the overview. Zooming and panning enable an analyst to restrict attention to a particular area of the graph, which is visualized in the center of the display (Figure 1(E)). NetEx enables the analyst to explore data at the desired level of detail (i.e., multi-level analysis) without losing the big picture. Thus, the tool has the capability of being orienting even when a very small area is selected for focus in the detail view (overview + detail $^{38}$ ).

NetEx promotes interactive visual exploration, allowing users to select a particular element of the graph, such as a single node or a set of nodes, and to access thematic data not directly exposed in the network structure. A bubble link connects a node in the network and a pop-up data box showing its thematic data, a time-series plot of sensor readings in the case of electrical power networks. ${ }^{\dagger}$ The thematic data is attached to the network view with a line of bubbles which anchors the data box to the node it is related to (Figure 1(A)). The use of bubbles instead of solid lines distinguishes between graph edges (solid lines) and anchors (bubble lines). This feature, which can be seen as an extension of probes proposed earlier $^{28,41}$ to graphs, should be useful to any system that has more than one level of representation.

There is clear similarity between NetEx and DragMag, ${ }^{41}$ a tool that supports zooming in on different parts of an image using several zoom windows. Each DragMag zoom window, which corresponds to a thematic data box in NetEx, is visibly connected to a zoomed-in patch in a base image, which corresponds to the NetEx network view. DragMag probes are solid lines while NetEx probes are lines of bubbles; we believe the bubbles provide a helpful contrast to the solid lines representing graph edges in the NetEx network view.

\subsection{Multi-focus Network Analysis: Multiple Nodes with Thematic Data}

Many analysis tasks—including fault diagnosis—require analysts to inspect and compare multiple nodes, with their thematic data sets, in order to make sense of what is happening in the network under study. By supporting the simultaneous opening and comparison of multiple thematic data sets, such as sensor reading time series, for nodes from different parts of a network, NetEx enables what we call multi-focus analysis.

NetEx simplifies comparison of different nodes, along with their thematic data, in several ways. The bubble lines supports the comparison of thematic data, such as time-series, for multiple nodes while at the same time connecting the thematic data to their respective locations in the graph structure. An analyst can also "merge" thematic data from different nodes into a single box by dragging a box and dropping it over another one (Drag \& Merge), as illustrated in Figure 2. This is particularly useful when the number of compared nodes is large. A different color is associated with each of the nodes to be compared. Both the line in the plots and the bubbles are painted with this color to allow for immediate association of nodes with corresponding data. Original colors are preserved in the merged box to help the analyst maintaining association between nodes and data, even when the latter is moved into a different box. Moreover, plots are lined up vertically in the side panel and a trace-line is displayed across all of them to help identifying events occurring at the same time for different nodes (Figure 1(B)). NetEx also allows the analyst to change the axis scale of data boxes so that a common scale is adopted.

\section{VISUALIZATION AND ANALYSIS OF NETWORKS: CASE STUDIES}

We have applied NetEx in several case studies. In a first case study, discussed in Section 4.1, we diagnose faults in an electrical power network. Here, we use data from ADAPT, ${ }^{45}$ a real-world electrical power network. We compare NetEx with a baseline tool; this comparison is the basis for the formal user study in Section 5. In a second case study, NetEx is applied to a social network built from the Enron e-mail data set. ${ }^{37}$ Section 4.2 shows how NetEx helps detect two key players in the company, namely Jeff Dasovich and Bill Williams, that were not previously known to the analysts using NetEx. A third case study shows how the techniques described in this paper allow better interactive analysis of Bayesian networks, ${ }^{1}$ especially comparison of conditional probability tables within them. ${ }^{43,44}$

\footnotetext{
${ }^{\dagger}$ NetEx provides, for the data boxes, both a completely separate visualization as well as floating diagrams. We experimented and found pros and cons of both approaches, and therefore ended up providing both options in NetEx.
} 


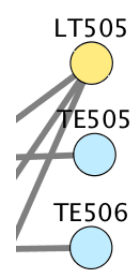

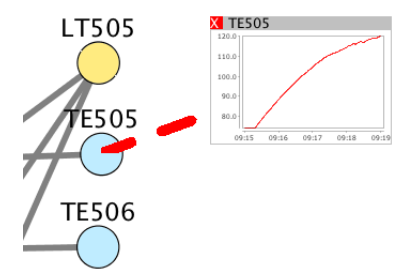

(b)

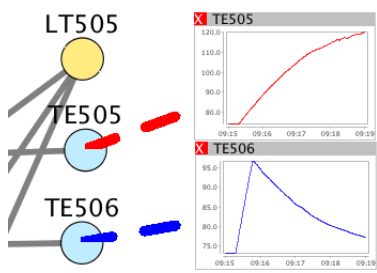

(c)

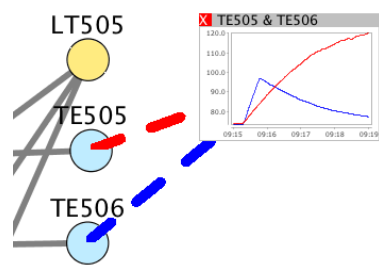

(d)

Figure 2. Alternative NetEx displays of a node-link graph in combination with thematic data, here time-series graphs, shown in data boxes, illustrated using the ADAPT electrical power network dataset: (a) no data box is shown; (b) the box of TE505 is shown; (c) the box of TE506 is also shown; (d) the two boxes are merged. Original colors are preserved for graphs in (d) to help the analyst maintaining association between nodes and data, even when the latter is moved into a different box.

\subsection{Diagnosing Faults in Electrical Networks}

This section describes the use of NetEx to diagnose faults in a real-world electrical power network, known as ADAPT, ${ }^{45}$ which has capabilities for power storage, distribution, and consumption. ADAPT contains batteries, electromechanical relays, circuit breakers, and different kinds of loads, such as pumps, fans, and light bulbs. Several sensors are available, measuring voltage, current, relay position, temperature, light intensity, and liquid flow. We consider scenarios taken from the ADAPT testbed, more specifically Tier 2 of DX 2009 competition dataset. ${ }^{\ddagger}$ These scenarios consist of nominal runs, where faults were not injected into ADAPT, as well as faulty runs involving one or more faults in components or sensors.

\subsubsection{Fault Diagnosis Examples}

We are interested in diagnosing which component(s) broke and at what time it (they) broke. By examining and comparing time series for a selected set of sensors (voltage, current, temperature, etc.), it is possible to diagnose a faulty scenario. Consider a case in which light bulb LGT400 breaks, stopping its emission of light and heat and drawing no current. Current sensor readings upstream of the broken component will show an abrupt drop, and the light (LT500) and temperature (TE500) sensors attached to the bulb will clearly show abnormal behavior (Figures 1 and 3). Specifically, a sudden drop of light intensity can be seen for LT500, while a sharp inversion of trend (from slowly increasing to decreasing) is observed for TE500. Other sensors on the same circuit, such as TE501 and TE502, show the expected slowly increasing behavior. This suggests that the problem must be related to only one load, LGT400.

We now discuss how the fault isolation task, which can involve several steps including scrolling, zooming, and multistep comparisons, is supported by the interactive visualization tools Intelliviz and NetEx.

\subsubsection{Baseline Visualization Tool: Intelliviz}

For the purpose of comparison, we used Intelliviz, ${ }^{\S}$ an existing visualization tailor-made for the ADAPT electrical power network. The tool provides two windows: a network window (Figure 3(B)), and a sensor window showing sensor readings (Figure 3(A)). There is no link between the windows, so that a plot can only be associated with the corresponding network node based on the common name. Plots of sensor readings are organized alphabetically. Plots corresponding to nodes which are very close in the network and interacting with each other may be very far apart in the sensor window and hard to compare. Since the ADAPT network contains about 170 nodes, half of which are sensors, the sensor window must be scrolled down several times in order to see all the plots. Finally, no overview nor zooming of the network are provided.

In order to perform the fault isolation task with this tool, the following steps are necessary:

1. Search for irregularities in sensor time series. Since the number and location of components and sensors involved in faults are unknown, all plots must be analyzed, but due to limited screen space it is not possible to look at all plots at the same time. ${ }^{\text {Il }}$ Also, there is no way to mark interesting plots, such as LT500 and TE500 in Figure 3(A). The analyst is thus required to remember (or manually write on the side) the names of the fault candidate nodes.

\footnotetext{
\#ttp://www.dx-competition.org/

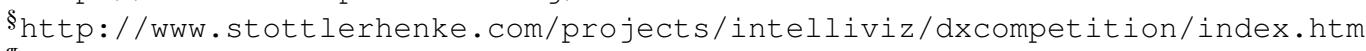

IThis is a general point, and applies not only to Intelliviz.
} 


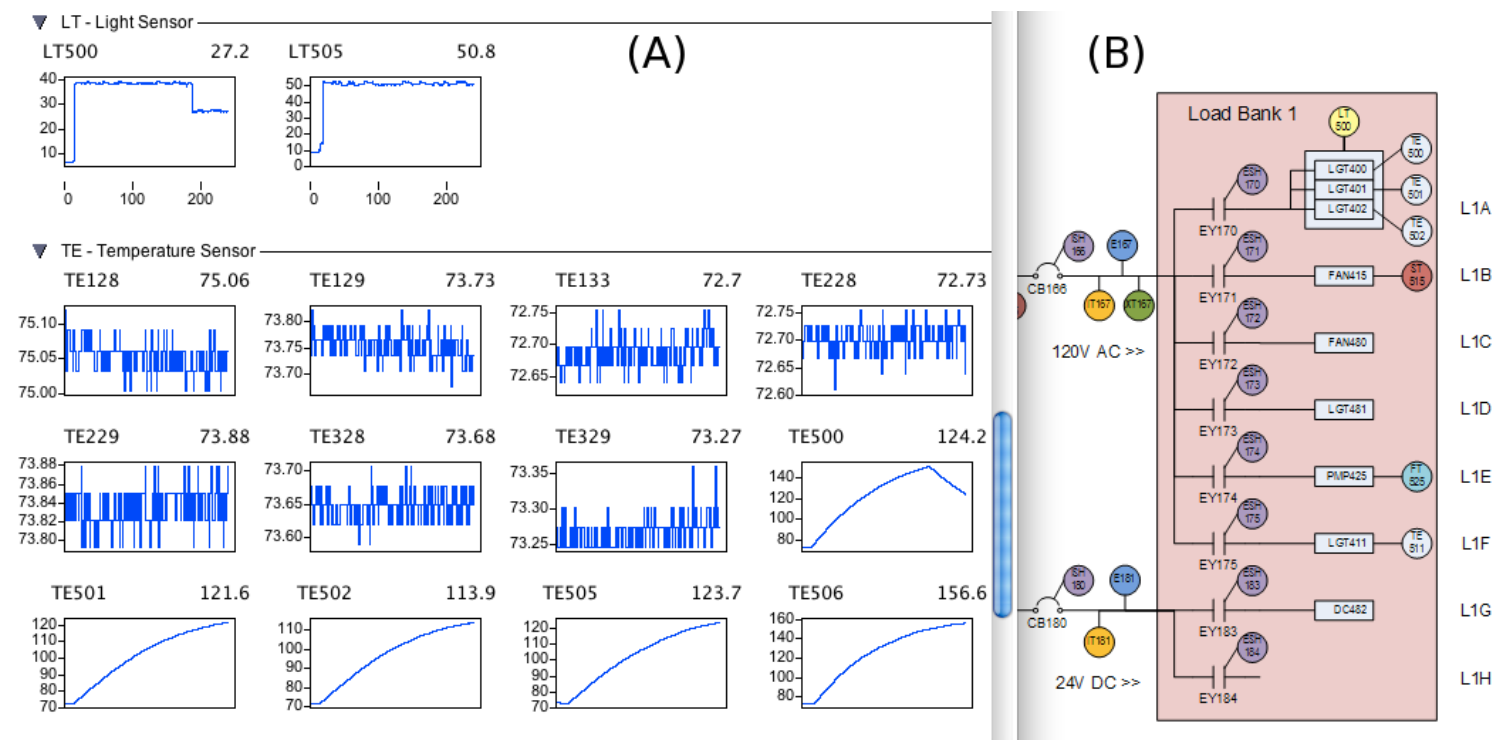

Figure 3. Intelliviz, a visualization tool for the ADAPT electrical power network. Two windows are provided: sensor readings window, with time series plots, shown to the left in the foreground (A) and network window shown to the right in the background (B).

\begin{tabular}{|c|c|c|}
\hline Diagnosis Step & Intelliviz & NetEx \\
\hline$\frac{\text { 1) Search for irregularities }}{\text { in sensor time series }}$ & $\begin{array}{l}\text { - Cannot mark interesting plots } \\
\text { - All plots automatically shown }\end{array}$ & $\begin{array}{l}\text { - Plot creation takes time and effort } \\
\text { - Interesting plots can be kept }\end{array}$ \\
\hline 2) Detect fault candidate nodes & $\begin{array}{l}\text { - Plots potentially hard to } \\
\text { compare due to ordering } \\
\text { according to type and name } \\
\text { - No visual link between thematic } \\
\text { plots and nodes }\end{array}$ & $\begin{array}{l}\text { - Plots easy to compare thanks } \\
\text { to box stacking, Drag \& Merge, } \\
\text { and common scale } \\
\text { - Bubbles visually link } \\
\text { thematic plots and nodes }\end{array}$ \\
\hline 3) Isolate faulty nodes & $\begin{array}{l}\text { - Requires switching between } \\
\text { sensor and network windows } \\
\text { - Harder due to lack of preparation } \\
\text { in search and detect steps }\end{array}$ & $\begin{array}{l}\text { - No need to switch between } \\
\text { different windows } \\
\text { - Easier thanks to preparations } \\
\text { made in search and detect steps }\end{array}$ \\
\hline
\end{tabular}

Table 1. Comparison between Intelliviz and NetEx with regards to each step of a diagnosis task. NetEx allows for thematic plots of interest to be selected and shown together in the same view, anchoring data to respective nodes through bubbles. During the first step, NetEx sets an analyst up for the second and third steps, and there are no similar capabilities in the baseline tool, Intelliviz.

2. Detect fault candidate nodes in the network. Since there is no link between the plots and the network view, fault detection requires the analyst to match sensor plots (Figure 3(A)) with network nodes (Figure 3(B)) based on component names only. Due to lack of explicit links (such as the bubble anchors of NetEx), this implies in the worst case a visual inspection of all nodes in the network. Many panning operations could be necessary, in addition to many switches between the network and sensor windows.

3. Isolate faulty nodes. The location of faulty components and sensors can be inferred by carefully analyzing irregularities (sudden current drop, slowly decreasing temperature, etc.), taking into account the relationships between candidate nodes in the circuit (current nodes on the same path as faulty light bulb LGT400, sensors LT500 and TE500 connected to LGT400). In general, this step also requires panning and switching between the network and sensor windows.

\subsubsection{Novel Visualization Tool: NetEx}

Our hypothesis is that NetEx, by supporting analysts in comparing readings from multiple sensors and quickly identifying interesting parts of the network, may provide strong support for fault diagnosis. Assuming that the side panel is used to display thematic data, NetEx enables an analyst to perform the same steps as Intelliviz, but in a different way:

1. Search for irregularities in sensor time series. The analyst first selects a group of nodes (Figure 1(D)) with one cursor drag, and then displays the corresponding thematic sensor plots in the side panel (Figure 1(B)) with one key stroke. Plots corresponding to candidate nodes can be kept in the side panel, making the following steps straightforward. For 


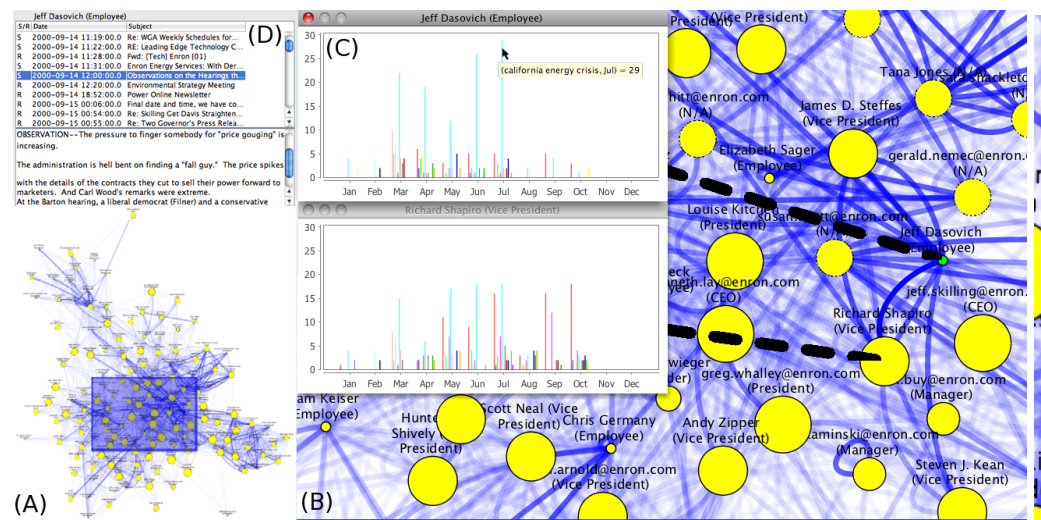

(a) NetEx network overview

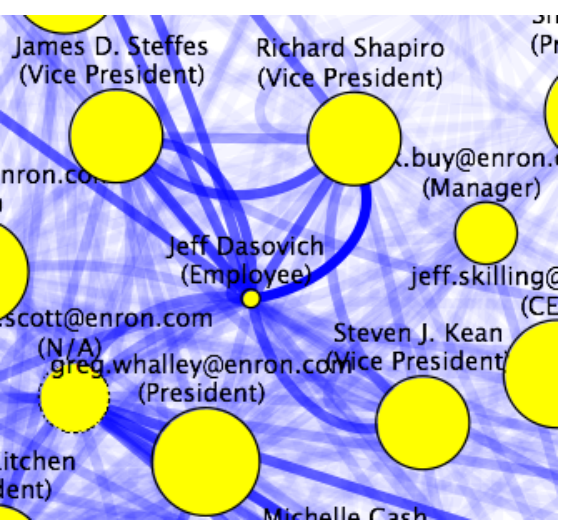

(b) NetEx detailed view

Figure 4. Discovery of Jeff Dasovich in Enron data set: a small node (low level employee) with several dark edges (high number of e-mails) to large nodes (high-level employees), representing an interesting area for further investigation. (a) Multi-focus and multi-level representation, in NetEx, for Enron social network: (A) overview level, (B) detail level, (C) thematic data level, and (D) datum level. Anchoring the thematic data level to the network view with large dashed bubbles allows low-level focused analysis and comparison while preserving the structure of the network. (b) Zooming in on Dasovich (node in center), we see how the high number of e-mail messages, exchanged between the employee Dasovich and the two vice presidents Steffes and Shapiro, clearly stands out in our visualization.

example, let us consider Figure 1, where the analyst has selected and expanded a number of nodes. After visual inspection of thematic data, an analyst may realize that only LT500, TE500 and IT167 are abnormal. The analyst might thus remove from the side panel the node boxes related to all other sensors, as they are no longer relevant to fault diagnosis.

2. Detect fault candidate nodes in the network. Bubble links enable immediate matching of candidate plots in thematic data boxes with the corresponding nodes (Figure 1(A)). No visual search for specific node names in the network, nor switching between different windows, is needed.

3. Isolate faulty nodes. Comparison of data is relatively easy in this case because (i) only interesting plots are shown in the side panel, and (ii) data from different nodes can be merged in the same box (Figure 2). There is no need to switch between different windows thanks to the visual link (bubbles) between nodes and their thematic data. Consider again Figure 1: after irrelevant thematic data boxes are removed, bubble lines enable an analyst to quickly realize that all interesting sensors (LT500, TE500 and IT167) are located on the same path in the electrical power network.

\subsubsection{Comparison of NetEx and Intelliviz}

Both Intelliviz and NetEx support electrical power system analysis and problem solving. Specifically, we discussed above how three problem solving steps of diagnosis—search, detect, and isolate-are supported by both these software tools. The different capabilities of each tool, with respect to the three problem solving steps, are summarized in Table 1.

\subsection{Social Network Visualization}

NetEx has been applied to visualize and analyze the Enron data set. ${ }^{37}$ Consisting of 250,000 e-mail messages exchanged between 150 Enron employees over a period of about 3 years, this corpus has been widely used for demonstrating knowledge discovery and visualization techniques. NetEx enabled an analysts to detect two key players, namely Jeff Dasovich and Bill Williams, that to our knowledge had not previously been identified using other visualization tools.

Focusing on Dasovich, NetEx shows interesting areas of the graph that are very clearly distinguishable at a glance, even if the whole network is displayed (see the small green node to the middle right of Figure 4(a)(A)). "By using the overview+detail capability of NetEx, the analyst's attention was captured by a very small node (i.e. marginal role in the organization) with (i) an impressive number of exchanged messages (i.e. very dark edges), (ii) a significant number of edges, and (iii) connections with large nodes (i.e. prominent roles). Zooming and panning gives a more detailed view of this node, corresponding to the employee Jeff Dasovich, and its neighbors (see Figure 4(a)(B)). Now it was quite easy to

\footnotetext{
${ }^{\|}$With a significantly larger network than Enron, in terms of nodes, edges, or both, interesting areas may not be so clearly distinguishable, and initial search or filter steps could be necessary. Such search or filter steps are not currently provided by NetEx.
} 
realize that there is a significant interaction between Dasovich and two vice presidents, namely James Steffes and Richard Shapiro. As pointed out by Pathak and Srivastava, ${ }^{52}$ the degree of interaction between these people is relatively high compared to the average in the network. There are also concealed ${ }^{* *}$ relations, which may be of great interest to analysts since they may hide malicious activities.

We can get more information about the topics discussed by Dasovich in his e-mails by activating the thematic data box, another multi-level capability of NetEx, for his node. As Figure 4(a)(C) shows, California Energy Crisis is the dominant topic of Dasovich's messages, especially in the summer of 2001, just before the Enron scandal broke. This information might be very relevant for analysts interested in identifying the main actors involved. The concealed nature of some of these relationships can be investigated in detail using the NetEx message viewer (Figure 4(a)(D)).

\section{USER STUDY: DIAGNOSING FAULTS IN ELECTRICAL NETWORKS}

In order to test the performance of NetEx, we conducted a user study in the area of debugging electrical networks (see Section 4.1). In this section we report on the objective and subjective results of this user study.

\subsection{Subjects, Tutorials, and Tasks}

We asked 25 subjects to use the software tools Intelliviz and NetEx to diagnose faults in the ADAPT electrical power network. Subjects were graduate students at the CMU Silicon Valley campus, with at least some knowledge about electrical circuits. Subjects (23 males and 2 females) were in the age range 20-30 years and volunteered to participate in the study with no cash compensation. Before an experiment, we guided a subject through a tutorial presenting the ADAPT electrical power network and the visualization tools. We also guided subjects through one tutorial task per tool, similar to the discussion in Section 4.1, to show how Intelliviz and NetEx can be used for fault diagnosis.

During training, each subject learned the tools by going through two instructive tutorials taking approximately 30 minutes. Tutorial 1 is an example of a fault occurring to a load (FAN415). Tutorial 2 is an example of a fault occurring to a connecting component (CB136)..$^{\dagger \dagger}$ Each of the tutorial tasks aligns better to one of the experimental tasks, but since all users used both tools (as further discussed below), we believe there is no or little bias. Subjects were instructed to look at the time series plots showing sensor readings to find out which component(s) or sensor(s) failed, and at what time it happened, with a hard time limit of 20 minutes per scenario. Subjects were not given any particular indication about preferring speed or accuracy.

We considered two different scenarios (Task 1 and Task 2), each with two concurrent failures. Subjects were randomly placed into two groups; 13 people in Group 1 and 12 in Group 2. Each subject used both Intelliviz and NetEx. Subjects in Group 1 first used Intelliviz for Task 1, and then NetEx for Task 2. Subjects in Group 2 first used NetEx for Task 1, and then Intelliviz for Task 2.

Task 1 consisted of a scenario with two faulty loads (light bulbs LGT406 and LGT407). The faults can be detected, see Figure 5(a), by noticing the drop of temperature reported by the sensors attached to the loads (TE506 and TE507), the drop of light intensity reported by the sensor LT505, and the drop of current in the path from the battery to the loads (sensors IT261, IT267, and IT340). This task was intended to be relatively easy, as the faults are quite similar, there was very little ambiguity in the evidence, and a similar example had been shown during the tutorial.

Task 2 consisted of a scenario with a faulty circuit breaker (CB180) and a faulty sensor (ESH160A). As illustrated in Figure 5(b), the circuit breaker fault can be detected by noticing the change of state, from closed to open, reported by the sensor attached to the circuit breaker (ISH180) and noticing the drop of current and power downstream of the circuit breaker (IT181 and E181). The issue with the sensor could be identified by noticing that all the other components downstream of the relay were working fine. In other words, the problem was the sensor itself, not the relay. This task was intended to be harder than Task 1, due to the possible confusion from having two concurrent faults of different nature on the same path.

\footnotetext{
${ }^{* *}$ Consider, for example, the following situation: $X$ sends an e-mail to $Y$, with carbon copy (Cc) to $Z$ and blind carbon copy (Bcc) to $B$. Consequently, everybody involved knows that $X$ communicated with $Y$ and $Z$, but only $X$ and $B$ know that the e-mail was also sent to $B$. The relation between $X$ and $B$ is said to be concealed because others (in particular $Y$ and $Z$ ) are not aware of it, due to the use of Bcc.

${ }^{\dagger}$ Tutorial 1 (Exp_792_pb_t2), Tutorial 2 (Exp_758_pb_t2), Task 1 (Exp_814_pb_t2), and Task2 (Exp_766_pb_t2ff) are available on-line at http://www.stottlerhenke.com/projects/intelliviz/dxcompetition/datamontage/ competition/index_t2.htm.
} 


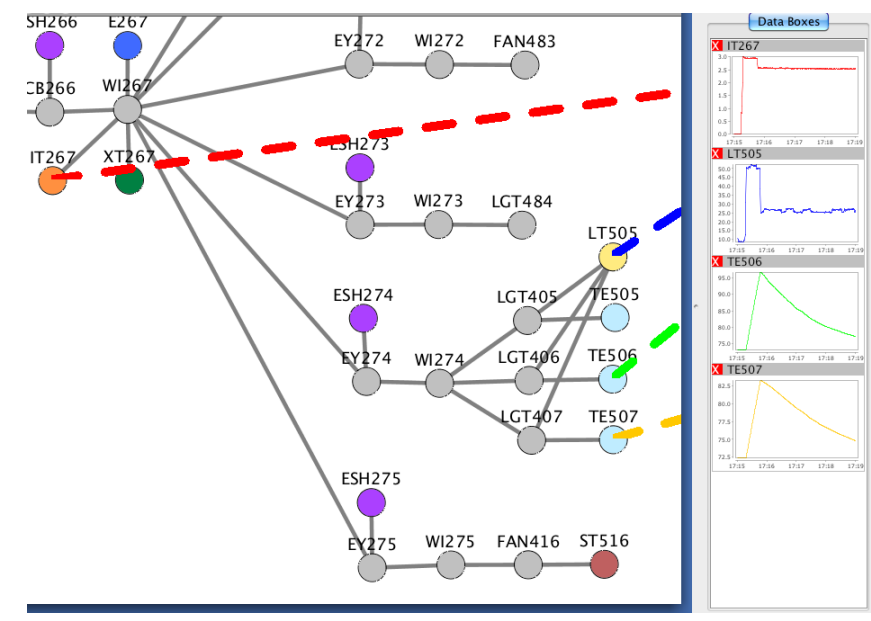

(a)

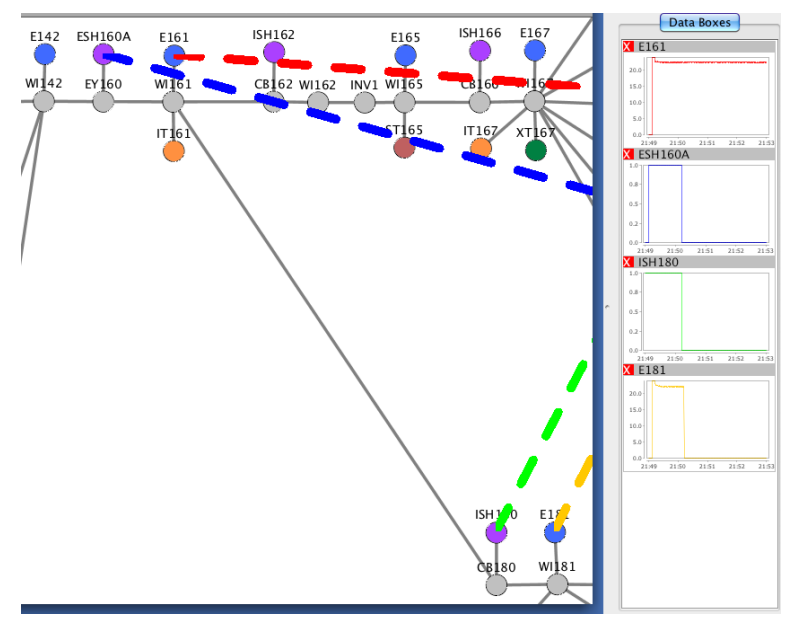

(b)

Figure 5. Key NetEx plots for experimental tasks. (a) Task 1: the drop of temperature reported by sensors TE506 and TE507, the drop of light intensity reported by sensor LT505, and the drop of current reported by sensor IT267 indicate that light bulbs LGT406 and LGT407 are very likely to be faulty. (b) Task 2: the change of state reported by sensor ISH180 and the drop of power reported by sensor E181 indicate that circuit breaker CB180 is very likely to be faulty. The change of state reported by sensor ESH160A is not supported by a drop of power in sensor E161, suggesting that the sensor itself might be faulty in this case.

\subsection{Answers and Analysis}

Answers from subjects were recorded both during the experiments with Intelliviz and NetEx as well as afterwards. During an experiment, for each detected fault, subjects were asked to report the faulty component or sensor, along with the time when the fault had originally happened, and a short explanation of their answer. We also recorded the time when they detected a fault; no feedback was provided to the subject as to whether the fault they had found was correct or not. The answers were then analyzed as discussed below; our results are presented in Section 5.3. After the experiment, subjects were asked to answer a post-task questionnaire, providing their experience around the tasks and tools. These answers were then analyzed as discussed below, leading to the results presented in Section 5.4 and Section 5.5.

We used two objective metrics to analyze users' performance on each task: the $F_{1}$ score to measure accuracy and the diagnosis time to measure speed. The $F_{1}$ score is defined as: $F_{1}=\frac{2 \times T P}{2 \times T P+F P+F N}$, where $T P$ is the number of correctly identified faults (true positives), $F P$ is the number of false alarms (false positives), and $F N$ is the number of missed faults (false negatives). The $F_{1}$ score measures the accuracy of a subject's answer, taking into account both correct diagnoses and mistakes. The best $F_{1}$-value is 1 , meaning no mistakes $(F P+F N=0)$, while the worst $F_{1}$-value is 0 , meaning no correct diagnoses $(T P=0)$.

In five cases, the subject's brief explanation for a diagnosis did not agree with the reported fault. We determined that subjects accidentally confused the name of a component with the name of the attached sensor, and gave preference to the explanation. Moreover, subjects who obtained an $F_{1}=0$ score for a task were not considered in the following analysis for that task. ${ }^{+}$This happened for one subject in Task 1 and six subjects in Task 2.

\subsection{Objective Results}

Figure 6(a) summarizes the results of subjects using Intelliviz and NetEx on Task 1 (simple task) and Task 2 (complex task). The time subjects used to finish a task is summarized on the $x$-axis, while the accuracy is summarized on the $y$-axis. Averages and standard deviations for both dimensions are shown. Indeed, the task difficulty was about right, as most of the subjects took much of the allocated time to finish Task 2. For both Task 1 and Task 2, the results suggest that NetEx is more accurate while Intelliviz is faster. This is especially prominent for Task 2 (the complex task).

The mean and standard deviation of the $F_{1}$ accuracy scores were as follows: On Task 1, the IntelliViz score was $0.92 \pm 0.16$ and the NetEx score was $1.00 \pm 0.00$. On Task 2, the IntelliViz score was $0.73 \pm 0.22$ and the NetEx score

\footnotetext{
$\$$ Note that no subjects abandoned the study. However, some subjects obtained a $F_{1}=0$ score, which we took to mean that they either did not fully understand the task or ran out of time before finding a meaningful answer to the task.
} 


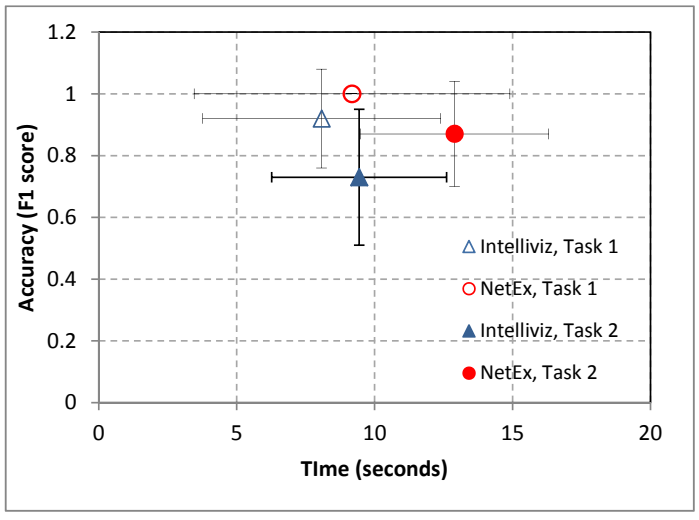

(a)

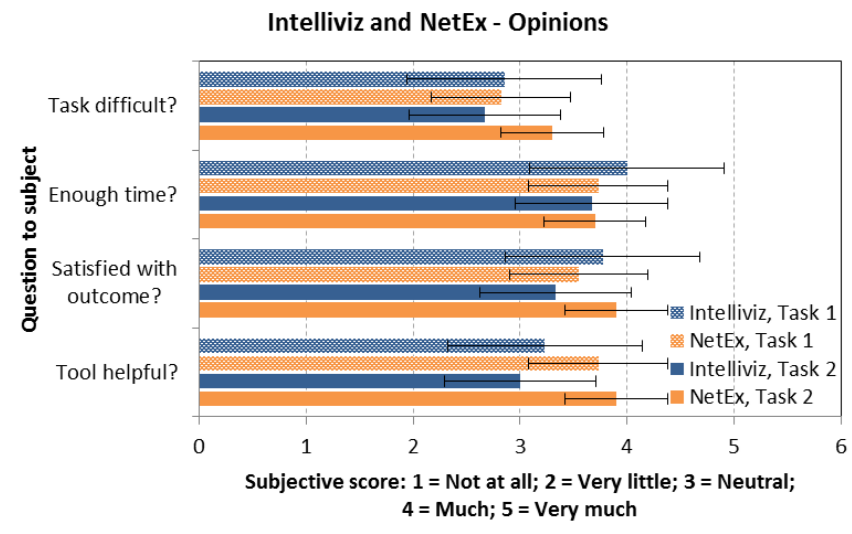

(b)

Figure 6. Comparison of Intelliviz and NetEx. (a) Performance in terms of time ( $x$-axis) and accuracy ( $y$-axis). Subjects were faster but less accurate in their diagnostic problem solving when they used Intelliviz. (b) Answers summarized from post-questionnaire. When using NetEx, subjects appropriately thought that Task 2 was more difficult than Task 1; there is no such difference for Intelliviz.

was $0.87 \pm 0.17$. For both tasks, subjects who used NetEx were on average more accurate than subjects who used Intelliviz. To analyze this statistically, a $t$-tests was used to test whether the mean for one group is greater than for the other group. For Task 1, NetEx was more accurate than Intelliviz with $p=0.061$; there was no significant difference between the completion times. For Task 2, Intelliviz was faster than NetEx with $p=0.018$; however NetEx was more accurate than Intelliviz with $p=0.075$.

One interpretation of the above result is that NetEx enables more accurate results thanks to its key multi-level and multi-focus capabilities: the bubble anchors, the side panel, and the capability of showing and hiding plots on demand. The combination of these features enables fast visual association of candidate thematic plots with the corresponding nodes in the network. This is particularly crucial for Task 2, where the interaction between the faulty components requires more than one step of the analysis.

The mean and the standard deviation of the diagnosis times, in minutes, were as follows: On Task 1, the time for IntelliViz was $8.08 \pm 4.31$ and the time for NetEx was 9.18 \pm 5.72 . On Task 2, the time for IntelliViz was $9.44 \pm 3.17$ and the time for NetEx was $12.90 \pm 3.41$. Subjects who used Intelliviz were on average significantly faster than subjects who used NetEx on the more difficult Task $2(p=0.018)$; this is not the case for Task $1(p=0.298)$.

We have two hypotheses for why Intelliviz users were faster, on average, than NetEx users. First, some subjects using Intelliviz quit very quickly (perhaps thinking, typically incorrectly, that they had solved the diagnosis problem) but in fact did not perform very accurately compared to subjects using NetEx. This hypothesis is supported by the lower accuracy score for Intelliviz, see Figure 6(a). Second, Intelliviz shows all plots simultaneously in the sensor window, and in addition this Intelliviz window can host a larger number of plots than the NetEx side panel in a single view (approximately 20 compared to approximately 5). This second point suggests that the search step will generally be faster in Intelliviz than in NetEx. However, NetEx users are supported by the more interactive and scalable approach of being able to select a group of nodes they want to inspect and to execute another action (key stroke) to see the thematic plots.

\subsection{Subjective Results: Intelliviz versus NetEx}

In one part of the post-questionnaire, we asked the same questions about the experimental system NetEx and the control Intelliviz. Figure 6(b) summarizes the results, in terms of averages and standard deviations (error bars) of four postexperiment questions. Note that exactly the same questions were asked about Intelliviz and NetEx. While there is substantial overlap between the error bars and means in many cases, several interesting trends emerge. For Task difficult?, only for NetEx is there a (correct) indication that Task 2 (the complex diagnosis task) was harder. Using a $t$-test, we found

We realize that the use of $p$-values is somewhat controversial and emphasize that when $0.1>p>0.05$ this is often considered "weakly significant" or a "trend." In this paper, we include the specific $p$-value for $p<0.1$, or show error bars, and avoid the use of descriptive terms (like "strongly significant" and "weakly significant"). When $p \geq 0.1$, we say "not significant" or give the $p$-value. 


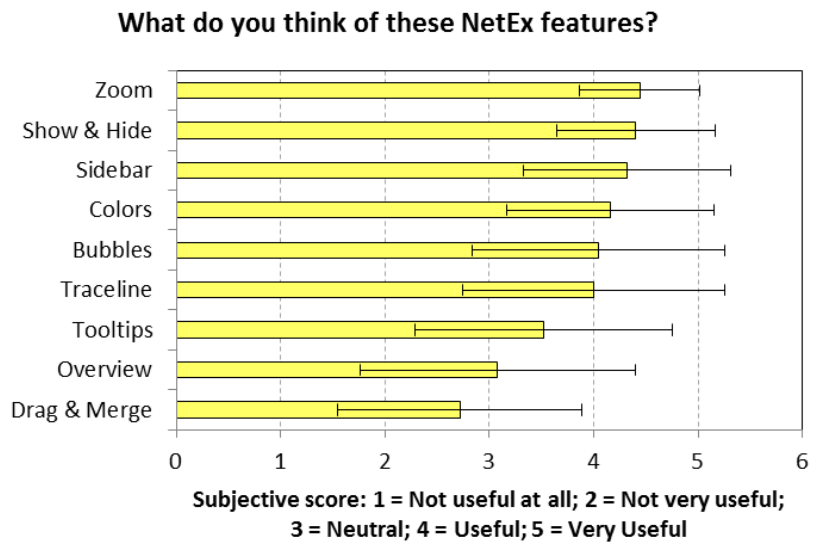

(a)
Do you think the features in NetEx were confusing?

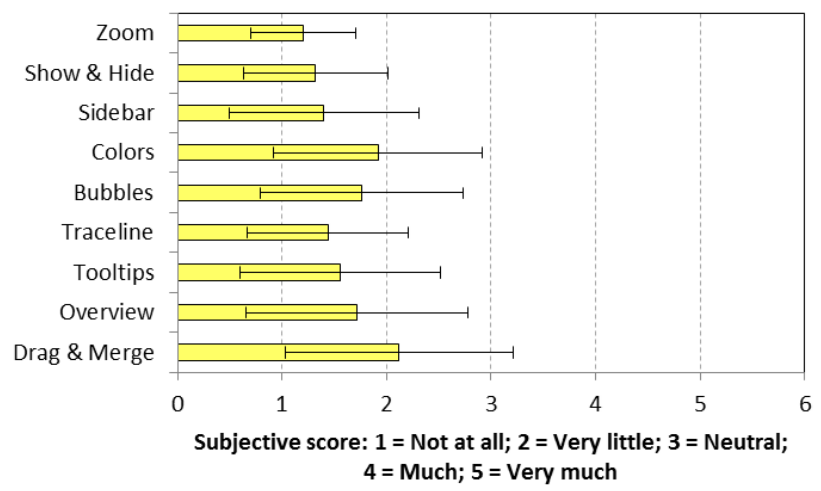

(b)

Figure 7. Opinions about different features of NetEx, summarized from post-questionnaires. Only the features Drag \& Merge and possibly Overview were seen as not useful-see panel (a); no features were seen as confusing-see panel (b).

in Task 2 that the Task difficult? score for NetEx is higher than that for Intelliviz (with $p=0.060$ ). For Satisfied with outcome?, there appears to be a difference in outcome satisfaction in favor of NetEx only for the complex task. Using a $t$-test, the score for NetEx is for Task 2 greater than that for Intelliviz (with $p=0.053$ ). For Tool helpful?, there appears to be a bigger gap between NetEx and Intelliviz for Task 2, in favor of NetEx. Using a $t$-test, in Task 2 the score on the Tool helpful? question for NetEx is greater (better) than the score of Intelliviz, with $p=0.019$.

\subsection{Subjective Results: Opinions about NetEx}

Figure 7(a) and Figure 7(b) show results, in terms of averages and standard deviations (error bars), for two post-experiment questions asked about nine different features of NetEx: Bubbles, Colors, Drag \& Merge, Overview, Show \& Hide, Sidebar, Tooltips, Traceline, and Zoom. Drag \& Merge is illustrated in Figure 2. Show \& Hide refers to one of the multi-focus and multi-level techniques in NetEx, namely how users can open and close thematic data boxes (time series plots) for each node according to their needs. Tooltips show some information about nodes. For example, in the ADAPT network, tooltips show the name and type of a sensor ("E161 (Voltage)", "C112 (Current)", ... ). In the Enron network, the name and position of a person is shown ("Frank Ermis (Director)", ...). The remaining six features are illustrated in Figure 1.

Figure 7(a), showing results for the question What do you think of these NetEx features?, is ordered according to average usefulness, with the most useful feature at the top. The only NetEx feature with an average score worse than (i.e., less than) Neutral is Drag \& Merge. We ran a statistical hypothesis test, specifically a one-population $t$-test, with the null hypothesis that $\mu=\mu_{0}=3.0$ (since Neutral $=3.0$ ). All results are (in terms of usefulness of NetEx features) significantly away from Neutral $=3.0$, except for Drag \& Merge and Overview.

We hypothesize that the reasons for these NetEx features being less useful include the following. Subjects did not use Drag \& Merge very much; they may have been able to perform the tasks just by comparing plots (taking advantage of the Trace-line). Perhaps this feature was not required to solve the experimental tasks, or perhaps learnability was a reason this feature was not used much. Drag \& Merge may not have been emphasized enough during the tutorial compared to other features such as Bubbles. Also, it was not possible to split plots that had been merged together by means of Drag \& Merge, and this might have prevented subjects from using this feature as much as we had hoped for. As for Overview, we now think that the experimental ADAPT network was not large enough to fully test the usefulness of this feature.

Figure 7(b) is structured essentially the same way as Figure 7(a), except we here focus on results for the question Do you think the features in NetEx were confusing? Interestingly, all averages are better than (less than) Neutral and statistically significantly away from Neutral $=3.0$. This suggests that no NetEx features were, in general, confusing to users.

Using a one-population $t$-test, the level of significance was better than $\alpha=0.025$ for Tooltips and $\alpha=0.005$ or better for the remaining NetEx features (excluding Drag \& Merge and Overview).

Using a one-population $t$-test, the level of significance was $\alpha=0.005$ or better for all NetEx features. 


\subsection{Discussion}

Our results suggest that NetEx users perceived the task to be harder compared to Intelliviz users $(p=0.060)$; they were nevertheless (and correctly) more confident about their performance on the task $(p=0.053)$. Moreover, NetEx users were more satisfied with the tool $(p=0.019)$.

For the relatively small ADAPT graph, our results suggest that Intelliviz is faster than NetEx on the search step, because it automatically shows thematic time series data for all nodes. All the time series plots must be examined by an analyst and, at least for a relatively small network such as ADAPT, this can be done quickly when all plots are available in the same window, as it is in Intelliviz. Sadly, Intelliviz shows thematic data for all nodes and this approach does not scale. Also, for more complex tasks looking at all thematic data might be daunting, even for quite small networks. Indeed, for specific comparisons and for networks that are larger than ADAPT, NetEx may have an advantage as it allows for plots that need to be compared to be selected and shown together in the same view. More importantly, during the search step NetEx sets an analyst up for the detect and isolate steps (see summary in Table 1). Our results suggest that these steps can then be performed faster and more accurately using NetEx compared to Intelliviz.

\section{CONCLUSION AND FUTURE WORK}

The multi-focus and multi-level analysis techniques discussed in this paper enable an analyst to identify and compare multiple nodes of interest. While many visualizations focus on analysis, NetEx focuses on showing a visually recognizable view of a node-link structure, coupled with multi-focus display of thematic data for important nodes. This paper demonstrates how comparing multiple views in a visual interactive analysis tool can reduce errors when investigating and comparing thematic data, for the purpose of complex fault diagnosis. The multi-focus and multi-level techniques succeed by supporting simultaneous analysis of multiple parts of a dataset while giving the analyst different views of and easy access to the network's graph structure. Using data from an electrical power network, we showed in a user study how NetEx may improve the analytical task of diagnosis. Our results suggest that, compared to a baseline tool, NetEx enables more accurate isolation of faulty nodes in complex situations involving multiple faults.

In future work, we would like to borrow from Intelliviz by showing, in NetEx, all or selected plots in a larger side panel at the beginning of the analysis. The analyst could then remove all plots showing a nominal behavior, also taking advantage of the bubble connections, thus the ending up with a relatively small number of candidate plots. NetEx could also be strengthened by supporting larger networks and visualizing (parts of) networks along with multiple fisheye foci. We also expect to further evaluate these visual interfaces and interaction techniques with additional graph exploration tasks.

The size and complexity of network-related data continue to expand. The power and value of this work is that it is part of the quest for scalable interactive computing systems that keep an analyst aware of the whole while exploring the details. We call for creating tools that aid people's limited memory while simultaneously exploring and comparing multiple nodes or areas of interest in networks. We encourage other researchers to work, as we are, on improving interactive techniques for exploring large and complex problems.

\section{ACKNOWLEDGMENTS}

This material is based, in part, upon work supported by NSF grants CCF-0937044 and ECCS-0931978.

\section{REFERENCES}

[1] Pearl, J., [Probabilistic Reasoning in Intelligent Systems: Networks of Plausible Inference], Morgan Kaufmann, San Mateo, CA, USA (1988).

[2] Overbye, T. J. and Weber, J. D., "New Methods for the Visualization of Electric Power System Information," in [Proc. of InfoVis'00], 131-136 (Oct 2000).

[3] Wong, P. C., Schneider, K., Mackey, P., Foote, H., Jr., G. C., Guttromson, R., and Thomas, J., "A Novel Visualization Technique for Electric Power Grid Analytics," IEEE Transactions on Visualization and Computer Graphics 15(3), 410-423 (2009).

[4] Abello, J. and van Ham, F., "Matrix Zoom: A Visual Interface to Semi-External Graphs," in [Proc. of InfoVis'04], 183-190 (Oct 2004). 
[5] Abello, J., van Ham, F., and Krishnan, N., "ASK-GraphView: A Large Scale Graph Visualization System,” IEEE Transactions on Visualization and Computer Graphics 12(5), 669-676 (2006).

[6] Adar, E., "GUESS: A Language and Interface for Graph Exploration," in [Proc. of CHI'06], 791-800 (Apr 2006).

[7] Auber, D., "Tulip : A Huge Graph Visualisation Framework," in [Graph Drawing Softwares], Mutzel, P. and Jünger, M., eds., Mathematics and Visualization, 105-126, Springer-Verlag (2003).

[8] Perer, A. and Shneiderman, B., "Integrating Statistics and Visualization for Exploratory Power: From Long-Term Case Studies to Design Guidelines," IEEE Computer Graphics and Applications 29(3), 39-51 (2009).

[9] Henry, N., Fekete, J.-D., and Mcguffin, M. J., "Nodetrix: a Hybrid Visualization of Social Networks," IEEE Transactions on Visualization and Computer Graphics 13(6), 1302-1309 (2007).

[10] Holten, D., "Hierarchical Edge Bundles: Visualization of Adjacency Relations in Hierarchical Data," IEEE Transactions on Visualization and Computer Graphics 12(5), 741-748 (2006).

[11] Lamping, J., Rao, R., and Pirolli, P., "A Focus+Context Technique Based on Hyperbolic Geometry for Visualizing Large Hierarchies," in [Proc. of CHI'95], 401-408 (May 1995).

[12] Phan, D., Xiao, L., Yeh, R., Hanrahan, P., and Winograd, T., "Flow Map Layout," in [Proc. of InfoVis'05], 219-224 (Oct 2005).

[13] Wong, N., "EdgeLens: An Interactive Method for Managing Edge Congestion in Graphs," in [Proc. of InfoVis'03], 51-58 (Oct 2003).

[14] Faloutsos, C., McCurley, K. S., and Tomkins, A., "Fast Discovery of Connection Subgraphs," in [Proc. of KDD'04], $K D D$ '04, 118-127 (Aug 2004).

[15] Gansner, E., Koren, Y., and North, S., "Topological Fisheye Views for Visualizing Large Graphs," in [Proc. of InfoVis'04 ], 175-182 (Oct 2004).

[16] McGuffin, M. J. and Jurisica, I., "Interaction Techniques for Selecting and Manipulating Subgraphs in Network Visualizations," IEEE Transactions on Visualization and Computer Graphics 15(6), 937-944 (2009).

[17] Pietriga, E., Bau, O., and Appert, C., "Representation-Independent In-Place Magnification with Sigma Lenses," IEEE Transactions on Visualization and Computer Graphics 16(3), 455-467 (2010).

[18] Schaffer, D., Zuo, Z., Greenberg, S., Bartram, L., Dill, J., and Roseman, M., "Navigating Hierarchically Clustered Networks Through Fisheye and Full-Zoom Methods," ACM Transactions on Computer-Human Interaction 3(2), 162188 (1998).

[19] van Ham, F. and van Wijk, J. J., "Interactive Visualization of Small World Graphs," in [Proc. of InfoVis'04], 199-206 (Oct 2004).

[20] van Ham, F. and Perer, A., ““'Search, Show Context, Expand on Demand”: Supporting Large Graph Exploration with Degree-of-Interest," IEEE Transactions on Visualization and Computer Graphics 15(6), 953-960 (2009).

[21] Wong, P. C., Mackey, P., Cook, K. A., Rohrer, R. M., Foote, H., and Whiting, M. A., "A Multi-Level Middle-Out Cross-Zooming Approach for Large Graph Analytics," in [Proc. of VAST'09], 147-154 (Oct 2009).

[22] Bezerianos, A., Chevalier, F., Dragicevic, P., Elmqvist, N., and Fekete, J.-D., "GraphDice: A System for Exploring Multivariate Social Networks," in [Proc. of EuroVis'10], 863-872 (Jun 2010).

[23] Lee, B., Smith, G., Robertson, G. G., Czerwinski, M., and Tan, D. S., "FacetLens: Exposing Trends and Relationships to Support Sensemaking within Faceted Datasets," in [Proc. of CHI'09], 1293-1302 (Apr 2009).

[24] Lex, A., Streit, M., Partl, C., Kashofer, K., and Schmalstieg, D., "Comparative Analysis of Multidimensional, Quantitative Data," IEEE Transactions on Visualization and Computer Graphics 16(6), 1027-1035 (2010).

[25] Viau, C., McGuffin, M. J., Chiricota, Y., and Jurisica, I., "The FlowVizMenu and Parallel Scatterplot Matrix: Hybrid Multidimensional Visualizations for Network Exploration,” IEEE Transactions on Visualization and Computer Graphics 16(6), 1100-1108 (2010).

[26] Wattenberg, M., "Visual Exploration of Multivariate Graphs," in [Proc. of CHI'06], 811-819 (Apr 2006).

[27] Weaver, C., "Multidimensional Data Dissection Using Attribute Relationship Graphs," in [Proc. of VAST'10], 75-82 (Oct 2010).

[28] Butkiewicz, T., Dou, W., Wartell, Z., Ribarsky, W., and Chang, R., "Multi-Focused Geospatial Analysis Using Probes," IEEE Transactions on Visualization and Computer Graphics 14(6), 1165-1172 (2008).

[29] Elmqvist, N., Henry, N., Riche, Y., and Fekete, J.-D., "Mélange: Space Folding for Visual Exploration," IEEE Transactions on Visualization and Computer Graphics 16(3), 468-483 (2010). 
[30] Shoemaker, G. and Gutwin, C., "Supporting Multipoint Interaction in Visual Workspaces," in [Proc. of CHI'07], 999-1008 (Apr 2007).

[31] Baldonado, M. Q. W., Woodruff, A., and Kuchinsky, A., "Guidelines for Using Multiple Views in Information Visualization," in [Proc. of AVI'00], 110-119 (May 2000).

[32] Collins, C. and Carpendale, S., "VisLink: Revealing Relationships Amongst Visualizations," IEEE Transactions on Visualization and Computer Graphics 13(6), 1192-1199 (2007).

[33] Heer, J., "Exploring Enron." http://hci.stanford.edu/jheer/projects/enron (2005).

[34] Stasko, J., Gorg, C., and Liu, Z., "Jigsaw: Supporting Investigative Analysis through Interactive Visualization," Information Visualization 7(2), 118-132 (2008).

[35] Weaver, C., "Building Highly-Coordinated Visualizations in Improvise," in [Proc. of InfoVis'04], 159-166 (Oct 2004).

[36] von Landesberger, T., Kuijper, A., Schreck, T., Kohlhammer, J., van Wijk, J. J., Fekete, J.-D., and Fellner, D. W., "Visual Analysis of Large Graphs," in [Proc. of EG'10 - STARs], 37-60 (May 2010).

[37] Shetty, J. and Adibi, J., "The Enron Email Dataset Database Schema and Brief Statistical Report," tech. rep., University of Southern California (2004).

[38] Cockburn, A., Karlson, A., and Bederson, B. B., "A Review of Overview+Detail, Zooming, and Focus+Context Interfaces," ACM Computing Surveys 14(1), 1-31 (2008).

[39] Herman, I., Melancon, G., and Marshall, M. S., "Graph Visualization and Navigation in Information Visualization: A Survey," IEEE Transactions on Visualization and Computer Graphics 6(1), 24-43 (2000).

[40] Selker, T., "Style and Function of Graphic Tools," in [Proc. of GI'99], 123-131 (Jun 1999).

[41] Ware, C. and Lewis, M., "The DragMag image magnifier," in [Conference companion on Human factors in computing systems ], CHI-95, 407-408 (1995).

[42] Ghoniem, M., Luo, D., Yang, J., and Ribarsky, W., "Newslab: Exploratory broadcast news video analysis," in [Proc. of the 2007 IEEE Symposium on Visual Analytics Science and Technology], VAST-07, 123-130 (2007).

[43] Cossalter, M., Multi-focus Multi-level Network Visualization, Master's thesis, Carnegie Mellon University (Aug 2011).

[44] Cossalter, M., Mengshoel, O., and Selker, T., "Visualizing and Understanding Large-Scale Bayesian Networks," in [Proc. of the AAAI'11 Workshop on Scalable Integration of Analytics and Visualization], 12-20 (Aug 2011).

[45] Poll, S., Patterson-Hine, A., Camisa, J., Garcia, D., Hall, D., Lee, C., Mengshoel, O. J., Neukom, C., Nishikawa, D., Ossenfort, J., Sweet, A., Yentus, S., Roychoudhury, I., Daigle, M., Biswas, G., and Koutsoukos, X., "Advanced Diagnostics and Prognostics Testbed," in [Proc. of DX'07], 178-185 (May 2007).

[46] Russell, D. M., Stefik, M. J., Pirolli, P., and Card, S. K., "The cost structure of sensemaking," in [Proc. of INTERCHI'93, ACM Conference on Human factors in Computing Systems], 269-276 (1993).

[47] Heer, J. and Boyd, D., "Vizster: Visualizing Online Social Networks," in [Proc. of InfoVis'05], 32-39 (Oct 2005).

[48] Dunne, C., Shneiderman, B., Gove, R., Klavans, J., and Dorr, B., "Rapid understanding of scientific paper collections: Integrating statistics, text analytics, and visualization," JASIST: Journal of the American Society for Information Science and Technology (2012).

[49] Gove, R., Dunne, C., Shneiderman, B., Klavans, J., and Dorr, B., "Evaluating visual and statistical exploration of scientific literature networks," in [VL/HCC '11: Proc. 2011 IEEE Symposium on Visual Languages and HumanCentric Computing ], 217-224 (2011).

[50] Steinberger, M., Waldner, M., Streit, M., Lex, A., and Schmalstieg, D., "Context-preserving visual links," IEEE Transactions on Visualization and Computer Graphics 17, 2249-2258 (2011).

[51] Shannon, P., Markiel, A., Ozier, O., Baliga, N. S., Wang, J. T., Ramage, D., Amin, N., Schwikowski, B., and Ideker, T., "Cytoscape: A Software Environment for Integrated Models of Biomolecular Interaction Networks," Genome Research 13(11), 2498-2504 (2003).

[52] Pathak, N. and Srivastava, J., "Automatic extraction of concealed relations from email logs," in [International Workshop and Conference on Network Science], (May 2006). 